\title{
Extrinsic behaviour of PZT ceramics near the morphotropic phase transition
}

\author{
A. Albareda, R. Pérez, J. García, J. Tiana and E. Pérez \\ Department of Applied Physics. Universitat Politècnica Catalunya. \\ Campus Nord, Ed. B4, c/ Jordi Girona 1-3, 08034 Barcelona, Spain.
}

\begin{abstract}
The aim of this work is to study the dependence of the linear and non-linear dielectric, piezoelectric and mechanical coefficients on the Ti fraction in PZT ceramic compositions near the morphotropic phase boundary. The dependence of these coefficients with the defect concentration is also analysed. Special attention is given to the extrinsic piezoelectric measurements related to the domain wall movement. Since the non-linear behaviour is produced by the extrinsic phenomenon, the non-linear measurements provide a good extrinsic characterization of materials. In all cases the physical explanation of the behaviour is sought.
\end{abstract}

\section{INTRODUCTION}

The crystal structure near the PZT morphotropic phase boundary (MPB) changes with the composition of $\mathrm{Ti}-\mathrm{Zr}$ from rhombohedral (low Ti quantity) to tetragonal (high Ti) through a narrow monoclinic phase. All these PZT materials present domain structure with domain wall of $180^{\circ}$ and non- $180^{\circ}$ between the directions of the domain polarisation of adjacent domains. The variations in the crystal structure impose variations on the possible adjacent polarisation domain, because not all the combinations are possible.

The different type of polarisation between adjacent domains produces different effects when a domain wall movement is produced: the extrinsic effect. The $180^{\circ}$ domain wall contributes only to dielectric behaviour, while non- $180^{\circ}$ walls also contribute to the elastic compliance and piezoelectric coefficients. Whereas intrinsic behaviour is related to the deformation of the elemental cell, the extrinsic behaviour implies higher coefficients. The domain wall movement is also very dependent on the dopants. The type and the quantity of these impurities help to stop the domain growth.

All these effects imply not only high coefficients, but also dielectric, elastic and piezoelectric nonlinear behaviours [1-4]. Sometimes these effects are not wanted, for example when the losses increase in high power devices.

The aim of this paper is to study the influence of the composition and doping quantities on the properties of ceramics. Special attention is given to the non-linear properties that can provide information about the extrinsic behaviour produced by the domain wall movements.

The series material studied in this paper have compositions in the interval: $0.479<\mathrm{x}<0.495, \mathrm{x}=\mathrm{Ti}$ fraction of $\mathrm{PbZr}_{(1-\mathrm{x})} \mathrm{Ti}_{\mathrm{x}} \mathrm{O}_{3}(\mathrm{PZT})$. Two series of materials (made by Ferroperm Piezoceramics $\mathrm{A} / \mathrm{S}$ ) have been used with high $(\mathrm{H})$ or low $(\mathrm{L})$ acceptor dopants. 


\section{LINEAR CHARACTERIZATION}

The dielectric permittivity (real and imaginary) depends very much on the structure, and therefore on the $\mathrm{x}$ fraction of $\mathrm{Ti}$ in the PZT composition. When the composition is near the MPB there are more domain wall orientations, and thus a large surface domain wall with a low energy may remain. Fig. 1 shows that $\varepsilon_{33}$ increases with the $\mathrm{x}$ Ti fraction when it approaches the MPB. This plot also shows that high doped ceramics $(\mathrm{H})$ have higher dielectric constant than ceramics with low impurities $(\mathrm{L})$.

In Fig. 2, the plot of dielectric losses shows a minimum of losses for a composition near $\mathrm{x}=0.485$. However, the most significant aspect is the decrease of losses with the high defect concentration $(\mathrm{H})$. This effect could be explained if we consider that the movement of the domain wall is a bending, so it appears as reversible and without increasing the losses. The bending domain wall movement model implies a reversible commutation of dipoles near the wall domain when an electric field is applied, which returns to the original orientation when the electric field disappears.

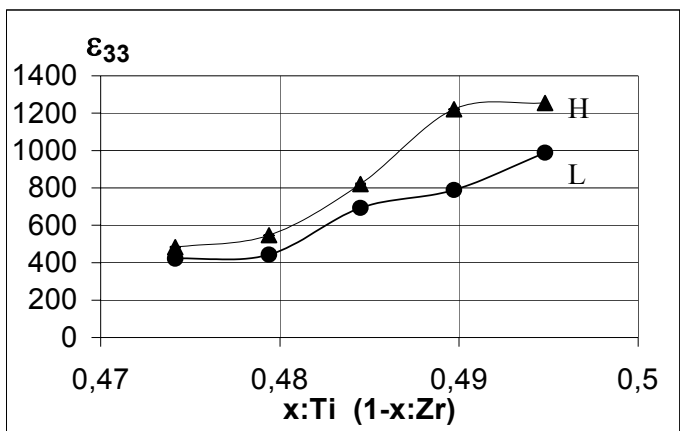

Figure 1. Dielectric constant versus $x$ : Ti fraction in PZT composition.

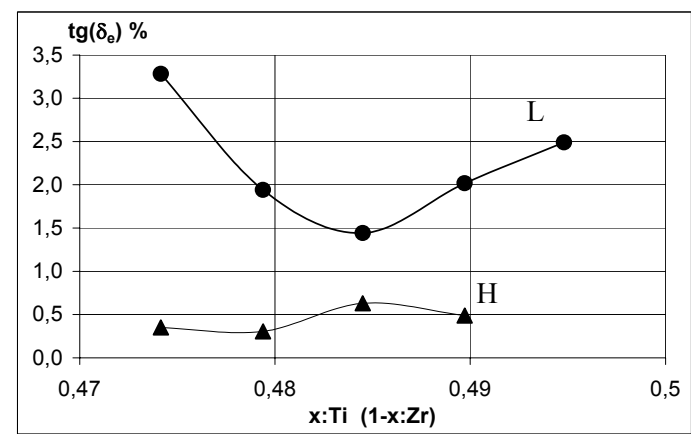

Figure 2. Dielectric losses versus $x$. (H) high and (L) low doping quantities.

The above behaviours are also observed in the mechanical losses, measured at resonance, Fig. 3: the losses are lower for high doped ceramics $(\mathrm{H})$.

Like the dielectric constant, the piezoelectric coefficient $\mathrm{d}_{31}$ increases with the $\mathrm{x}$ Ti fraction. Since this dependence is similar to the dielectric constant $\varepsilon_{33}$, Fig. 4 relates both coefficients. It is shown that there is an approximate linear relation between these coefficients, with a bigger piezoelectric coefficient for the ceramics with high $(\mathrm{H})$ doping acceptors. This result implies that the impurities favour the domain wall of non- $180^{\circ}$ more than the $180^{\circ}$, because the wall of non- $180^{\circ}$ increases not only the dielectric coefficient but also the piezoelectric one.

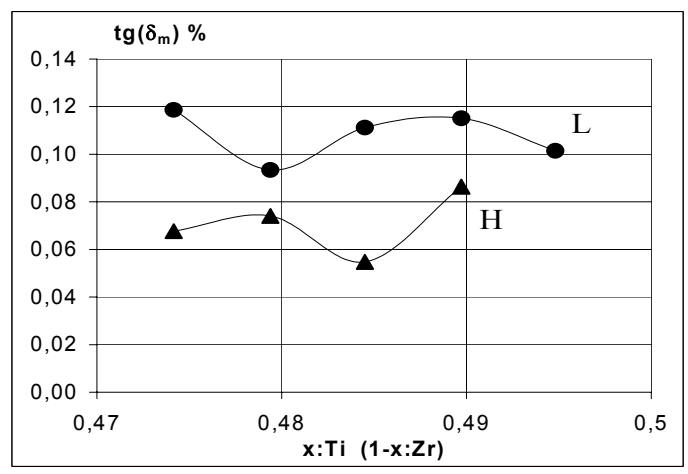

Figure 3. Mechanical losses versus $x$.

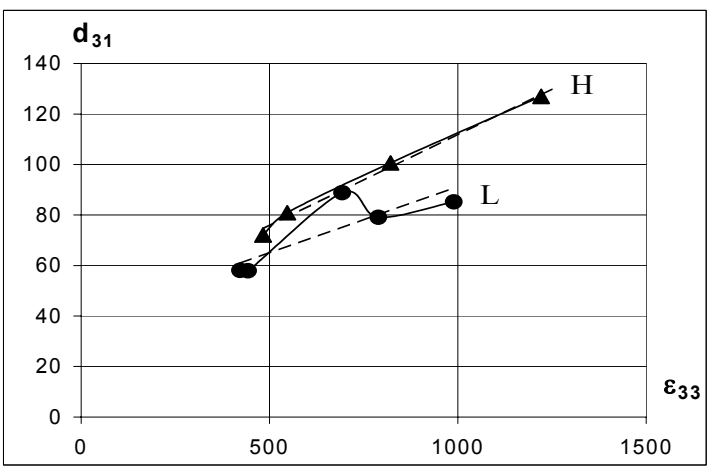

Figure 4. Piezoelectric $d_{31}$ coefficient versus dielectric constant. (--) Linear adjustment. 


\section{NON-LINEARITIES}

\subsection{Dielectric non-linearity}

The non-linear measurements are made at $1 \mathrm{kHz}$, by a modified comparison capacitance bridge that is compensated at low signal in order to obtain the non-linear contribution to the electric displacement $\mathrm{D}(\mathrm{E})$, and the non-linear dielectric constant increment $\Delta \varepsilon\left(\mathrm{E}, \mathrm{E}_{0}\right)$, at every instantaneous electric field, E. To prevent overheating of the ceramics due to the high signal excitation, burst signal excitation with only 5 cycles are used.

After the subtraction of the antisymmetric contribution $\varepsilon_{\mathrm{A}}$ (a low contribution), a discrimination is made between the irreversible contribution $\varepsilon_{\alpha}$, which corresponds to the dependence only on the electric field amplitude $\varepsilon_{\alpha}\left(\mathrm{E}_{0}\right)$, and the reversible contribution $\varepsilon_{\beta}\left(\mathrm{E}, \mathrm{E}_{0}\right)$, depending on the instantaneous electric field:

$$
\Delta \varepsilon\left(\mathrm{E}, \mathrm{E}_{0}\right)=\varepsilon_{\mathrm{A}}\left(\mathrm{E}, \mathrm{E}_{0}\right)+\varepsilon_{\alpha}\left(\mathrm{E}_{0}\right)+\varepsilon_{\beta}\left(\mathrm{E}, \mathrm{E}_{0}\right) .
$$

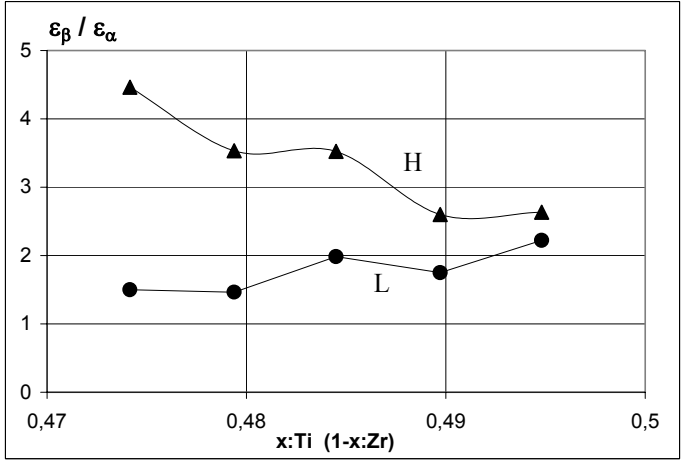

Figure 5. Quotient between the irreversible $\varepsilon_{\beta}$ and reversible $\varepsilon_{\alpha}$ non-linear dielectric constant, versus x.

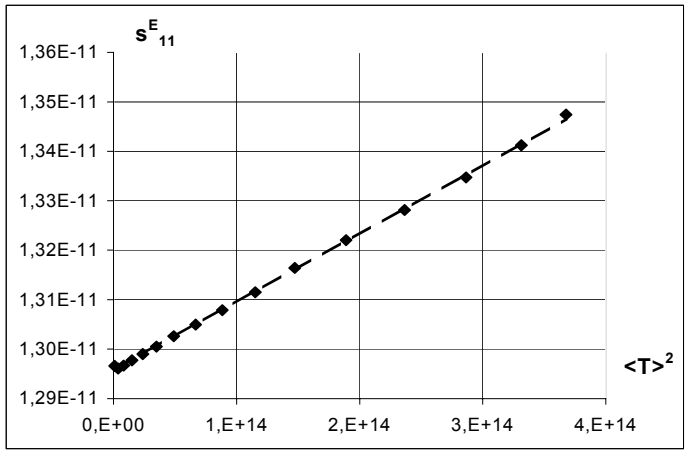

Figure 6. Non-linear compliance increase versus quadratic mean stress.

The measurements show a non-linear behaviour typical for hard piezoceramics: firstly, the irreversible contribution $\varepsilon_{\alpha}$ is proportional to the square of the electric field amplitude $\mathrm{E}_{0}^{2}$; secondly, the contribution of the reversible $\varepsilon_{\beta}$ is greater than the irreversible $\varepsilon_{\alpha}$ non-linearity, as can be observed in Fig. 5. Thus these materials have lower losses. This behaviour is greater for highly doped ceramics, and is very interesting for high power applications, because low losses and low non-linear losses are required for these devices. The bending effect, described below, could explain this low contribution to the losses.

\subsection{Mechanical non-linearities}

Measurements have been made at resonant frequency, with burst excitation. A laser vibrometer enables the velocity vibration measurement to be taken. The method used, described in [5], consists in the impedance measurements at constant frequency near the resonance. The increase of the real and imaginary part of impedance are related to the increases of losses $\Delta \operatorname{tg}\left(\delta_{\mathrm{m}}\right)$ and shift frequency, respectively, and thus to the compliance increase $\Delta \mathrm{s}^{\mathrm{E}}{ }_{11}$. The increase of the compliance has a quadratic dependence with the mean stress, $\Delta \mathrm{s}^{\mathrm{E}}{ }_{11}=\mathrm{c}<\mathrm{T}>^{2}$, Fig. 6 , as in the hard ceramics. This dependence is similar for the losses increase. 


\section{EXTRINSIC BEHAVIOUR}

It is assumed that the linear piezoelectric coefficient has both intrinsic and extrinsic contributions. It could be measured from the linear or low level values $e_{31 \text { lin }}=d_{31} / s^{E}{ }_{11}$ (Fig. 7). On the other hand the non-linearities are caused by the domain wall movement: the extrinsic contribution. From the previous non-linear measurements, it is possible to obtain the extrinsic piezoelectric behaviour. These nonlinear coefficients are generally different to the linear ones. The plot of $d_{31}$ versus $s_{11}^{E}$, Fig. 7, enables us to see the linear as well as the non-linear behaviour [4]: The extrinsic value is measured from the local slope $\mathrm{e}_{31 \text { ext }}=\Delta \mathrm{d}_{31} / \Delta \mathrm{s}_{11}^{\mathrm{E}}$, by the non-linear measurements.

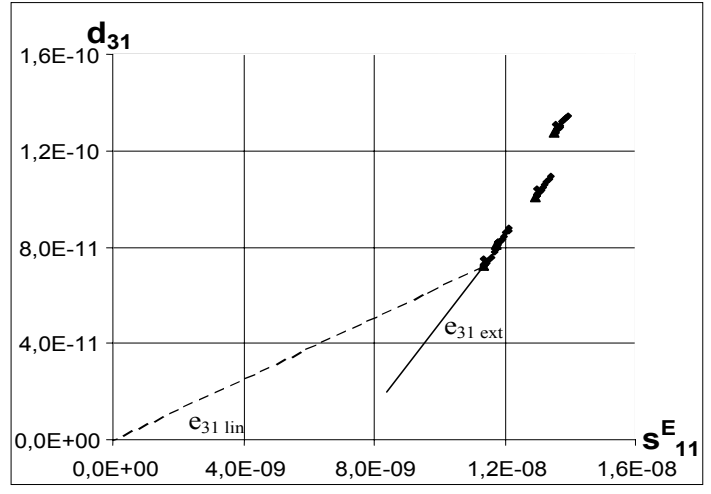

Figure 7. Piezoelectric $d_{31}$ versus compliance $\mathrm{s}^{\mathrm{E}}{ }_{11}$.

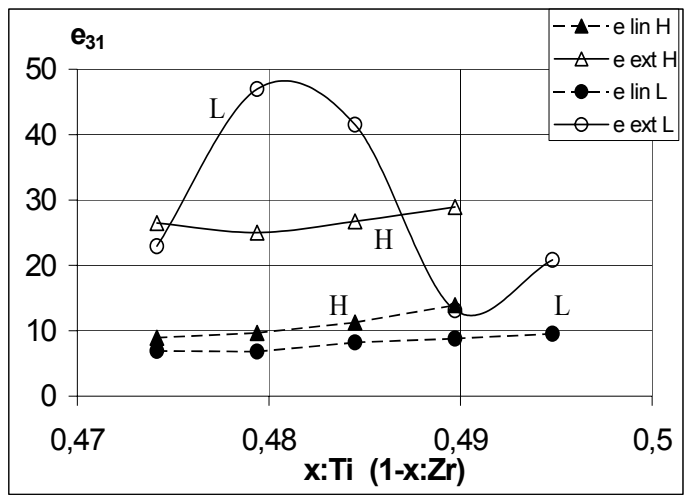

Figure 8. Extrinsic and linear piezoelectric $e_{31}$.

In all these ceramics, the extrinsic coefficient is higher than the linear one. Fig. 8 shows the linear and extrinsic values of $e_{31}$ piezoelectric coefficients for high and low doped ceramics. These measurements imply that for low doped ceramics (L) the extrinsic $e_{31}$ coefficient depends strongly on the $\mathrm{x}$ Ti fraction. For ceramics with high quantity of defects $(\mathrm{H})$, this dependence is very low, while the linear value is, for this series, higher than for the low doped ceramics (L).

\section{CONCLUSIONS}

The behaviour of PZT ceramics is strongly dependent on the crystal structure as well as on the defect concentration. Near the morphotropic phase boundary, MPB, all dielectric, piezoelectric and elastic coefficients increase. Due to the domain wall movement, the extrinsic contribution produces high dielectric and piezoelectric coefficients, and can be well characterized through the non-linear measurements.

Aknowledgments: All PZT ceramics have been supplied by Ferroperm Piezoceramics A/S, thanks to the collaboration of E. Ringgaard and W. Wolny. This work has been supported by the Spanish MEC (project MAT2004-01341) and the European Network POLECER (G5RT.CT-2001-05024).

\section{References}

1. D. Damjanovic, M. Denmartin. J. Phys.: Condens. Matter., 9 (1997) p. 4943.

2. D. Hall, P. Stevenson. Ferroelectrics, 228 (1999) p. 139.

3. J. García, R. Perez, A. Albareda. J Phys D: Appl. Phy., 34 (2001) p. 3279.

4. R. Perez, A. Albareda, J. García, J. Tiana, E. Ringgaard, W. Wolny. J Phys D: Appl. Phy., 37 (2004) p. 2648.

5. R. Perez, A. Albareda. J. Acoust. Soc. Am. 100 (1996) p. 3561. 